\title{
Using Commercial Activity Monitors to Measure Gait in Patients with Suspected iNPH: Implications for Ambulatory Monitoring
}

Shiv Gaglani ${ }^{1}$, Jessica Moore ${ }^{1}$, M Ryan Haynes ${ }^{1}$, Jamie B. Hoffberger ${ }^{1}$, Daniele Rigamonti ${ }^{1}$

1. Department of Neurosurgery, The Johns Hopkins University School of Medicine

$\square$ Corresponding author: Jessica Moore, jmoor110@jhmi.edu

Disclosures can be found in Additional Information at the end of the article

\section{Abstract}

Objectives: This study seeks to validate the use of activity monitors to detect and record gait abnormalities, potentially identifying patients with idiopathic normal pressure hydrocephalus (iNPH) prior to the onset of cognitive or urinary symptoms.

Methods: This study compared the step counts of four common activity monitors (Omron Step Counter HJ-113, New Lifestyles 2000, Nike Fuelband, and Fitbit Ultra) to an observed step count in 17 patients with confirmed iNPH.

Results: Of the four devices, the Fitbit Ultra (Fitbit, Inc., San Francisco, CA) provided the most accurate step count. The correlation with the observed step count was significantly higher $(p<0.009)$ for the Fitbit Ultra than for any of the other three devices.

Conclusions: These preliminary findings suggest that existing activity monitors have variable efficacy in the iNPH patient population and that the MEMS tri-axial accelerometer and algorithm of the Fitbit Ultra provides the most accurate gait measurements of the four devices tested.

Received 07/22/2015

Review began 07/27/2015

Review ended 11/17/2015

Published 11/17/2015

C) Copyright 2015

Gaglani et al. This is an open access article distributed under the terms of the Creative Commons Attribution License CC-BY 3.0., which permits unrestricted use, distribution, and reproduction in any medium, provided the original author and source are credited.
Categories: Neurology, Neurosurgery

Keywords: idiopathic normal pressure hydrocephalus, inph, activity monitor

\section{Introduction}

Patients with idiopathic normal pressure hydrocephalus (iNPH) commonly present with a triad of symptoms consisting of gait, cognitive, and/or urinary difficulties [1-2]. While patients can often retrospectively identify a gradual loss of functionality, current clinical monitoring cannot detect these subtle changes. Activity monitors present an opportunity for monitoring gait abnormalities in iNPH patients. These devices have been studied in the monitoring of gait abnormalities in patients with chronic diseases, including cerebral palsy, multiple sclerosis, Parkinson's disease, traumatic brain injury, stroke, rheumatoid arthritis, and osteoarthritis [39]. While these studies demonstrate the potential of activity monitors in clinical use and the need for ambulatory monitoring in many chronic conditions, they reported highly variable reliabilities in the gait monitoring of the activity monitors, concluding that the selection of an activity monitor must be application- and condition-specific [10-11]. In iNPH, monitoring the progression of symptoms in an ambulatory setting would require models of normal gait and the 
progression of iNPH gait changes.

The objectives of this study were to determine whether an activity monitoring device could accurately record gait in iNPH patients and, if so, which devices were most reliable in their step counts.

\section{Materials And Methods}

Seventeen patients with suspected iNPH based on cognitive and balance evaluations and MRI were enrolled to test the activity monitors. Activity monitors varied in the type of sensor, mounting location, and cost. The four monitors used were the Omron Step Counter HJ-113 (Omron Corp., Kyoto, Japan), New Lifestyles 2000 (NEW LIFESTYLES, Inc., Lee's Summit, MO), Nike Fuelband (Nike, Inc., Beaverton, OR), and Fitbit Ultra (Fitbit, Inc., San Francisco, CA). Patients wore the activity monitors on their right hip during two trials of a 10-meter walk. The trials were recorded on video. The step count was measured both by the pedometers and by two observers. The step counts measured by the observers were assessed for any differences and were reconciled by watching the video tape in the event of a disagreement. The correlation between the two step count values was calculated using linear regression. The correlation between the step count values of each device were calculated using bivariate correlation coefficient methods [12]. Step count variability between manual observation and device measurement was assessed using concordance correlation coefficients. Differences between measurements were plotted against average measurements using Bland-Altman plots.

Approval for this study was received from the Johns Hopkins Institutional Review Board (approval \#NA_00077065). Informed consent was obtained from each participant.

\section{Results}

The 17 patients ranged in age from 31 to 83 years, with a mean age of 69.7 years. Correlations between the step count values of the pedometer and observer were poor $\left(R^{2}<0.15\right)$ for the Omron Step Counter JH-113, New Lifestyles 2000, and Nike Fuelband. Correlation of the step count values was significantly higher for the Fitbit Ultra $\left(\mathrm{R}^{2}=0.57\right)$. The correlation of the observed step count value was significantly better with the step count of the Fitbit Ultra than with the Omron Step Counter HJ-113 (p<0.001), the New Lifestyles $2000(p<0.001)$, and the Nike Fuelband ( $p=0.009)$. Concordance correlation coefficients (CCC) between the device and manual step counts showed significant correlation only for the Fitbit Ultra (Table 1). The Bland-Altman plot of Fitbit versus manual step count showed a trend towards larger differences at higher step counts, but agreement at lower step counts (Figure 1). Similar plots for Omron, New Lifestyles, and Nike Fuelband activity monitors (not shown) showed relatively constant levels of difference regardless of step count with no areas of precise agreement. 


\section{Cureus}

\begin{tabular}{|c|c|c|c|}
\hline Device & Rho & $95 \% \mathrm{Cl}$ & Count Difference, Manual-Device (95\% Cl) \\
\hline Fitbit Ultra & 0.72 & $0.56,0.89$ & $2.74(-14.0,19.5)$ \\
\hline Omron & 0.19 & $-0.14,0.53$ & $7.0(-25.8,39.7)$ \\
\hline New Lifestyles & -0.27 & $-0.56,0.02$ & $8.4(-30.5,47.4)$ \\
\hline Nike Fuelband & -0.08 & $-0.29,0.12$ & $14.9(-7.3,37.1)$ \\
\hline
\end{tabular}

TABLE 1: Concordance Correlation Coefficients for Activity Monitor and Manual Step Counts

(A)

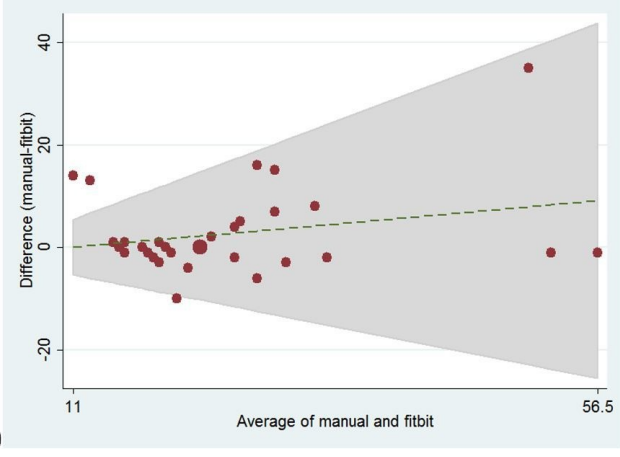

(B)

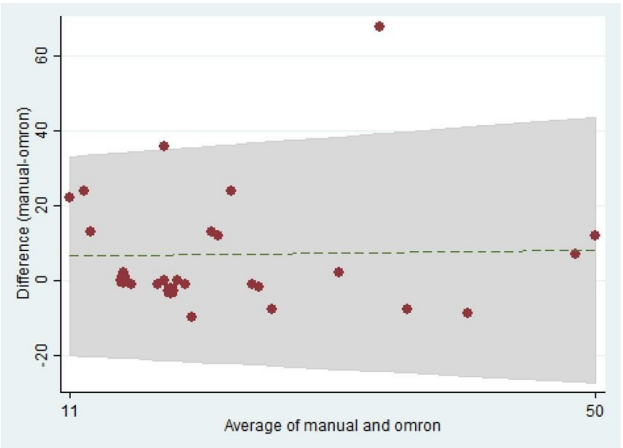

FIGURE 1: Bland-Altman Plot of Manual vs. (A) Fitbit Step

Count or (B) Omron Step Count

\section{Discussion}

While the accuracy of different activity monitors has been explored for a variety of neurological diseases, the use of activity monitors in the iNPH population has not been previously explored. Preliminary findings suggest that commercial activity monitors have a variable efficacy in the iNPH patient population. Of the four devices tested, the MEMS triaxial accelerometer and algorithm of the Fitbit Ultra was the most accurate at recording step counts in patients with iNPH. The step count measured by the Fitbit Ultra had a significantly higher correlation with the observed step count than any of the other three devices tested, as well as a significant, positive concordance correlation coefficient. However, the accuracy of the Fitbit Ultra was still relatively low, suggesting that the existing algorithms for accelerometers may be insufficient to measure the gait changes in iNPH. Further testing is required to assess other gait characteristics, such as step length, step height, and balance, as markers of neurological deterioration. Given the chronic nature of iNPH and the benefit from early detection of symptom progression, though, we believe that activity monitors still possess great potential to improve the quality of life of patients with iNPH.

Limitations of this study include the small study population and the limited number of devices tested. Furthermore, while this study provides preliminary data about the accuracy of different activity monitors, further testing is required to determine the accuracy of monitors in detecting 
gait changes over time, as this would determine the clinical utility of such devices. In addition, this study was unable to account for the degree of abnormality in a particular patient's gait. Therefore, the accuracy of different monitors at different severities of gait abnormalities could not be assessed.

Despite these limitations, this data provides a first step into applying activity monitors to the management of this chronic disease. The variable accuracy of activity monitors in the iNPH population is in keeping with studies of activity monitor accuracy in patients with other neurological diseases. These findings support the need for further testing of specific devices prior to their integration into a chronic disease care model.

The utility of activity monitors in iNPH monitoring and management depends on whether clinically relevant data may be collected. This will require longer-term monitoring of iNPH patients in the ambulatory setting, including monitoring of patients one-week pre- and oneweek post-lumbar puncture. If clinically relevant data can be collected, ambulatory monitoring may greatly benefit patients with iNPH and other chronic disorders in the future by offering healthcare providers information about the daily function and trends in symptoms.

\section{Conclusions}

As activity monitors become increasingly common, healthcare providers should seize the opportunity to engage patients in their own health maintenance [13]. This is particularly true for patients with chronic disease, about whom information about the daily function and longterm changes may be difficult to obtain. In this preliminary study, the Fitbit Ultra activity monitor proved to be the most accurate in monitoring the steps of patients with idiopathic normal pressure hydrocephalus, although even the accuracy of the Fitbit was limited $\left(\mathrm{R}^{2}=0.56\right.$, $\mathrm{CCC}=0.76$ ). Further studies are needed to illuminate whether activity monitors can identify early changes in patients with iNPH, allowing them to obtain treatment before significant symptom progression and impairment.

\section{Additional Information}

\section{Disclosures}

Human subjects: Consent was obtained by all participants in this study. Johns Hopkins University IRB issued approval NA_00077065. Animal subjects: All authors have confirmed that this study did not involve animal subjects or tissue. Conflicts of interest: In compliance with the ICMJE uniform disclosure form, all authors declare the following: Payment/services info: All authors have declared that no financial support was received from any organization for the submitted work. Financial relationships: All authors have declared that they have no financial relationships at present or within the previous three years with any organizations that might have an interest in the submitted work. Other relationships: All authors have declared that there are no other relationships or activities that could appear to have influenced the submitted work.

\section{References}

1. Shrinivasan A, Brandt-Pearce M, Barth A, Lach J: Analysis of gait in patients with normal pressure hydrocephalus. Proceedings of the First ACM Workshop on Mobile Systems, Applications, and. Services for Healthcare. ACM, Seattle, Washington; 2011. 1-6. 10.1145/2064942.2064947

2. Verny M, Berrut G: Diagnosis of normal pressure hydrocephalus in elderly patients: A review (Article in French). Geriatr Psychol Neuropsychiatr Vieil. 2012, 10:415-25. 10.1684/pnv.2012.0373

3. Backhouse MR, Hensor EM, White D, Keenan AM, Helliwell PS, Redmond AC: Concurrent 
validation of activity monitors in patients with rheumatoid arthritis. Clin Biomech (Bristol, Avon). 2013, 28:473-79. 10.1016/j.clinbiomech.2013.02.009

4. Fulk GD, Combs SA, Danks KA, Nirider CD, Raja B, Reisman DS: Accuracy of 2 activity monitors in detecting steps in people with stroke and traumatic brain injury. Phys Ther. 2014, 94:222-29. 10.2522/ptj.20120525

5. Hermann A, Ried-Larsen M, Jensen AK, Holst R, Andersen LB, Overgaard S, Holsgaard-Larsen A: Low validity of the Sensewear Pro3 activity monitor compared to indirect calorimetry during simulated free living in patients with osteoarthritis of the hip. BMC Musculoskelet Disord. 2014, 15:43. 10.1186/1471-2474-15-43

6. Iluz T, Gazit E, Herman T, Sprecher E, Brozgol M, Giladi N, Mirelman A, Hausdorff JM: Automated detection of missteps during community ambulation in patients with Parkinson's disease: A new approach for quantifying fall risk in the community setting. J Neuroeng Rehabil. 2014, 11:48. 10.1186/1743-0003-11-48

7. McAloon MT, Hutchins S, Twiste M, Jones R, Forchtner S: Validation of the activPAL activity monitor in children with hemiplegic gait patterns resultant from cerebral palsy. Prosthet Orthot Int. 2014, 38:393-99. 10.1177/0309364613506911

8. Sandroff BM, Motl RW, Pilutti LA, Learmonth YC, Ensari I, Dlugonski D, Klaren RE, Balantrapu S, Riskin BJ: Accuracy of StepWatch ${ }^{\mathrm{TM}}$ and ActiGraph accelerometers for measuring steps taken among persons with multiple sclerosis. PLoS ONE. 2014, 9:e93511. 10.1371/journal.pone.0093511

9. Yoneyama M, Kurihara Y, Watanabe K, Mitoma H: Accelerometry-based gait analysis and its application to Parkinson's disease assessment- part 2: a new measure for quantifying walking behavior. IEEE Trans Neural Syst Rehabil Eng. 2013, 21:999-1005.

10.1109/TNSRE.2013.2268251

10. Lützner C, Voigt H, Roeder I, Kirschner S, Lützner J: Placement makes a difference: Accuracy of an accelerometer in measuring step number and stair climbing. Gait Posture. 2014, 39:1126-32. 10.1016/j.gaitpost.2014.01.022

11. Morrow MMB, Hurd WJ, Fortune E, Lugade V, Kaufman KR: Accelerations of the waist and lower extremities over a range of gait velocities to aid in activity monitor selection for fieldbased studies. J Appl Biomech. 2014, 30:581-85. 10.1123/jab.2013-0264

12. Bivariate Correlation Comparisons. Accessed: December 2, 2014: http://psych.unl.edu/psycrs/statpage/biv_corr_comp_eg.pdf.

13. Case MA, Burwick HA, Volpp KG, Patel MS: Accuracy of smartphone applications and wearable devices for tracking physical activity data. JAMA. 2015, 313:625-26.

10.1001/jama.2014.17841 